Research Article

\title{
ESTIMATING BASE STATION-BASED INDOOR AND OUTDOOR ELECTRIC FIELD LEVELS BY ARTIFICIAL NEURAL NETWORKS
}

\section{Esin KARPAT ${ }^{1^{*}}$, Muhammed Rafet BAKCAN ${ }^{2}$, Mustafa Mohamedosman Abbaker IBRAHIM ${ }^{3}$, Berkant ÇELIK ${ }^{4}$, Ahmed Takieddine CHABBAR ${ }^{5}$, Ŏ̆uz DO $\breve{G} A N^{6}$}

The measurement of the electric field and the magnetic field is significant in order to determine electromagnetic pollution level compared to standards. In Turkey, electric field limit value, which is emitted by base station is 13.5 $V / m$ for one mobile communication operator. In addition, according to the regulation of medical devices, the limit of electric field value inside the hospital, where the medical devices are located, is $3 \mathrm{~V} / \mathrm{m}$. In this study, the measurement and evaluation of electromagnetic pollution inside and outside the Bursa Uludag University hospital building are performed and its compliance with national and international standards is examined. Moreover, the distribution of electric field in the environment is estimated by the artificial neural network and fuzzy logic methods considering the measurement results. The measured electric field values, estimated electric field values, and national standard values are compared.

Key words: Electromagnetic pollution, Electrical field, artificial neural network, fuzzy logic, prediction.

\section{Introduction}

In the shadow of these days, electromagnetic waves (EM) have become a complementary part of our every day life. This influences almost every aspect of our day-to-day life. However, the electromagnetic waves are radiated from natural, man-made sources such as TV, radio, wireless, Internet, cellular communication, and many other devices which are also exchanging increasing amounts of data by means of radio frequency (RF). So, this leads to installation of more base stations to cover new areas with new technology to work within the limited geographic area for unlimited

\footnotetext{
1 Department of Electrical and Electronics Engineering, Bursa Uludag University, Bursa, Turkey, (esinoz@uludag.edu.tr) https://orcid.org/0000-0002-2740-8183

2 Department of Electrical and Electronics Engineering, Bursa Uludag University, Bursa, Turkey, (muhammedrbakcan@outlook.com) (iD) https://orcid.org/0000-0002-9427-7371

3 Department of Electrical and Electronics Engineering, Bursa Uludag University, Bursa, Turkey, (Eng.mustafa1988s@gmail.com) iD https://orcid.org/0000-0003-1915-2349

4 Department of Electrical and Electronics Engineering, Bursa Uludag University, Bursa, Turkey, (berkantcelik@hotmail.com.tr) https://orcid.org/0000-0001-7457-5577

5 Department of Electrical and Electronics Engineering, Bursa Uludag University, Bursa, Turkey, ( $\underline{\text { ahmedchabbar@gmail.com) }} 10$ https://orcid.org/0000-0002-5919-399X

6 Department of Mechanical Engineering, Bursa Uludag University, Bursa, Turkey, (doganoguz@uludag.edu.tr) https://orcid.org/0000-0003-4203-8237
} 
users. That means the exposure of high levels of Electromagnetic Radiation (EMR) due to high RF power broadcasting in order to maximize the coverage of area [1-5].

Overall, EM waves, which have very wide range of frequencies, are part of non-ionizing radiation. While the operating system and frequencies can be in the range between $27 \mathrm{MHz}-300$ GHz. In the best case, in Europe and Turkey there is over $600 \mathrm{MHz}$ of spectrum available for mobile operators including the 800, 900, 1800, 2100 and $2600 \mathrm{MHz}$ Frequency Division Duplex (FDD) and Time Division Duplex (TDD) bands (GSM, CDMA, UMTS, LTE respectively, or a combination of generation technologies) [6,7]. The health effects of base stations and mobile phones in Turkey are really a tender topic. In gathering the accepted limits in many countries including European Union and the USA are recommended by The International Commission on Non-Ionizing Radiation Protection (ICNIRP), which is recognized by the World Health Organization (WHO). Turkey applies the limits which are determined in accordance with ICNIRP's limits [7]. Although, many parameters can change the EMR values such as the distance from base stations, the line of side path, the weather and Geographic nature and the number of traffic in the channel or the capacity of users in one cell. Thus, measurement and statistical evaluation have a large importance in the determination of EMR limits.

In the literature, there are many studies about the effect of high-frequency electromagnetic fields on human health. These studies were carried out with some animals, especially farm animals, as well as humans [8]. Research on humans has focused on children, adults and the elderly. High-frequency electromagnetic field was applied to tissues, such as skin, brain, etc. of children of up to 10 years old, and the resultant effects were compared with the results of adults. [9].

In addition, some studies have shown that children in the womb do not have cancer risk based on base station radiation [10]. In studies on children [11], the level of the electromagnetic pollution that the children between 1-10 years old are exposed to, due to wireless communication devices, are measured and Specific Absorption Rate (SAR) values of body tissues are compared with international standards [12].

In studies about sparrows, it was found that in the regions exposed to the frequencies of 900 $\mathrm{MHz}$ and $1800 \mathrm{MHz}$, the male sparrows were found to be less and this would have a negative effect on semination period [13].

Exposure of electromagnetic measurement in the GSM band studies is performed in regions where the population density is excessive. The mapping in the measured region is carried out and the results are compared with the international standards, determinations to determine the regions which have a negative effect on human health [14-20].

SAR values generated in case of close proximity to indoor devices, such as wireless baby monitors, phones, wireless headphones with different output powers, are calculated, the maximum and the average exposure Electromagnetic field (EMF) values are measured and evaluated [21].

The electromagnetic pollution, in the places where the mobile network is installed, can affect the communication of new networks. New methods are used to detect and reduce pollution [22].

In studies about the estimation of electromagnetic pollution in the literature, wireless electromagnetic field value detection in the indoor environment was estimated by Artificial Neural Networks (ANN) according to the position in the region. The attenuation effect of the facility obstacles between the base station and the mobile phone is estimated by artificial neural networks according to the mobile phone communication frequencies [23]. 
EMR has been obtained by artificial neural networks considering the parameters, such as building materials, window dimensions, and the number of windows in places such as houses, schools, and complex buildings [24].

The aim of this study is to measure and statistically analyse the EMR limits for GSM (2G\&3G) and LTE (4G\&4.5G). The electric field values on specific position from the base station were measured at different times of the days. The measurements were carried out at 45 different outdoor and 33 indoor locations in Bursa Uludag University's main campus and inside and outside the Faculty of Medicine Hospital. In this study, the predicted outdoor electromagnetic pollution values by artificial neural network and fuzzy logic are presented.

\section{Electromagnetic Wave Propagation}

Electromagnetic waves, which are the result of time-varying electric and magnetic fields, are means of energy or information. Typical electromagnetic waves are TV signals, radar rays, and radio waves. All electromagnetic waves have their signature features; they move at high speed and spread out from the source.

Electromagnetic waves, at distances sufficiently far from sources can be approximated as uniform plane waves. The $\mathrm{E}$ and $\mathrm{H}$ field components of these waves are everywhere normal to each other and to the direction of wave propagation [25].

The time-average power density carried by the electromagnetic wave can be calculated as

$$
\vec{P}_{\text {ave }}(z)=\frac{1}{T} \int_{0}^{T} \vec{P}(z, t) d t
$$

where; $\overrightarrow{\mathrm{P}}(\mathrm{z}, \mathrm{t})$ is the Poynting vector representing the instantaneous power density crossing unit area at a specific time in Eq.(2).

$$
\vec{P}(z, t)=\vec{E} x \vec{H}
$$

The time-average power density carried by the electromagnetic wave can be also calculated as

$$
\vec{P}_{\text {ave }}=\frac{1}{2} \operatorname{Re}\left\{\vec{E}_{S} x \vec{H}_{s}^{*}\right\}
$$

where $\overrightarrow{\mathrm{E}}_{\mathrm{S}}$ and $\overrightarrow{\mathrm{H}}_{\mathrm{S}}^{*}$ are the phasor forms of electric field and conjugate of magnetic field, respectively. The intrinsic impedance of the medium is calculated as

$$
Z_{0}=\frac{E_{0}}{H_{0}}
$$

In the far field region, where the electromagnetic waves are reffered to as radiative field, the electric field, magnetic field and power density are combined together with Eq. (5).

$$
P=\frac{E_{0}^{2}}{Z_{0}} \text { or } P=H_{0}^{2} Z_{0}
$$


where P ; Power density, $\left(\mathrm{Watt} / \mathrm{m}^{2}\right), \mathrm{E}_{0}$; Electric field intensity $(\mathrm{V} / \mathrm{m}), \mathrm{H}_{0}$; Magnetic field intensity $(\mathrm{A} / \mathrm{m})$ and $\mathrm{Z}_{\mathrm{o}}$; characteristic Impedance of free space, which is approximately $377 \Omega$ [26].

Eq. 5 shows that, in the far field, the measurement of the electric field or the magnetic field gives information about the electromagnetic level of the medium [25,27] and when the value of one of the field components is measured the other can be calculated.

\section{Measurement}

In this study, the electromagnetic pollution caused by the base station was measured in and around Bursa Uludag University medical school. The measurement results are divided into frequency ranges between $27 \mathrm{MHz}$ to $3 \mathrm{GHz}$, allocated by the ICTA and classified as GSM, UMTS, LTE and other frequencies other than these frequencies. Measurements were carried out inside and outside the hospital building.

The measurements outside the hospital were performed at the main entrance because of high density of people. The measurement points were selected as the places where the medical devices are located, the clinics close to the base station, the corridors and waiting rooms inside the hospital. The position and the measurement time are randomly selected and the most important selection criterion is determined to be the population density for indoor measurement. A total of 33 points were identified for indoor environment.

The measurement points in the front were close to the building and were across the base station. The measurements were carried out behind the windows and walls that face the base station.

In addition, measurements were performed inside the clinics and compared with national standards.

The measurement position and the measurement time are taken into consideration for outside measurement. A total of 45 measurements were identified for outdoor environment. After measurements, the data in the outdoor environment were obtained by taking the 6-minute average electric field values for each position considering the latitude and longitude of the measurement point and measurement time.

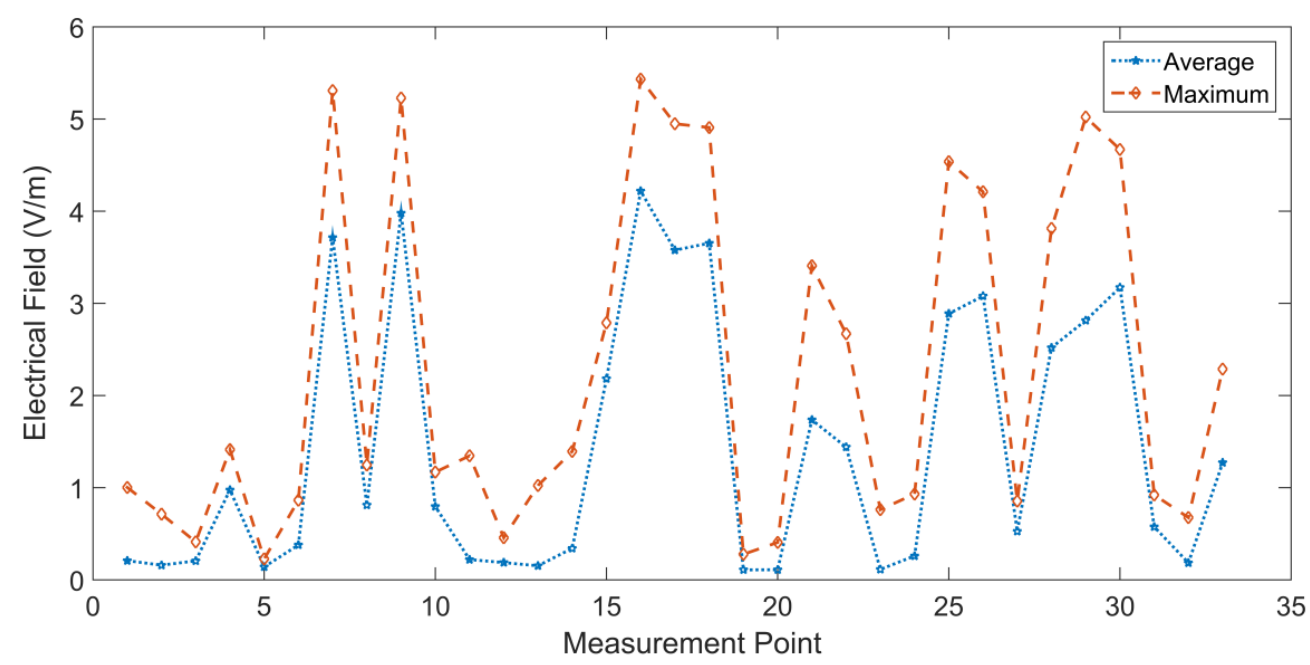

Figure 1. Indoor maximum and average electrical field value

The maximum value obtained indoor is $4.314 \mathrm{~V} / \mathrm{m}$ and the minimum electric field value is $0.108 \mathrm{~V} / \mathrm{m}$. The highest electric field value was detected on the 3rd floor of the building behind the window facing the base station. $0.97 \mathrm{~V} / \mathrm{m}$ value is measured behind the wall across the same base 
station. And the same value of the electric field $(0.97 \mathrm{~V} / \mathrm{m})$ is obtained when the measurement is repeated behind the wall in front of the same base station, on the same floor. Average and maximum peak electric field values obtained during indoor measurements are given in Fig.1.

\section{Artifical Neural Network}

The results of systematic measurement of outdoor measurements are trained by artificial neural network. The output electric field values are estimated based on the input parameters of the trained network. To estimate the total electric field value, there are 3 input parameters and 1 output value as shown in Fig. 2. The input parameters are latitude, longitude and measurement time values. In order to increase the accuracy of the predicted values given by the training network, it is necessary to select the input parameters as systematically as possible. If the random values are applied as input values, the MSE (mean squared error) value increases and the accuracy of prediction value decreases. For this reason, although the number of input data is not much, three different interrelated parameters are applied to the network as input variables.



Figure 2. Input parameters, measurement time, latitude, longitude for total E field prediction

In the second artificial neural network which was constructed in the study, different output parameters were tried to be estimated by using different inputs [28]. This created network has 5 inputs and 1 output parameters. Three of the input parameters are fixed and the other 2 input parameters are changed depending on the output parameter to be obtained. The parameters that are constant are latitude, longitude and measured time values of the measurement point, while the other 2 dependent input parameters are average electric field values covering the GSM, UMTS, LTE frequencies. One of the GSM, UMTS and LTE electric field values is estimated as an output parameter while the remaining 2 frequency components are defined as input parameters in Fig. 3. For example, in order to estimate the electric field value of UMTS frequency, input parameters are latitude, longitude, measurement time, GSM and LTE electric field values.

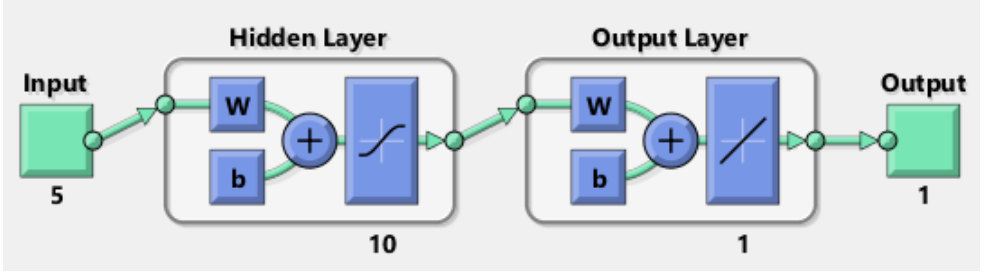

Figure 3. Input parameters; time, latitude, longitude, LTE and UMTS frequency values

In this way, the average electric field values of the other 2 frequency components are estimated using the same method. $70 \%$ of the measured data was used to train artificial neural networks, rest of 
$30 \%$ data were used for network test. There are 10 neurons in the hidden layer and one neuron in the output layer, in both prediction networks.

\section{Fuzzy logic}

Another prediction method used in the study is fuzzy logic [29]. In this method, the accuracy of the estimated results was increased. The range of input and output values was required for estimation. The input data were latitude and longitude values of the measurement point as in the ANN prediction model. However, the latitude values of the measurement points were rated between 0-400 and the longitude values were rated between 0-400 as shown in Eq. (6) and Eq. (7) respectively.

$$
\begin{aligned}
& L a t_{\text {out }}=\left(L a t_{\text {in }}-40.20696\right) * 10000 \\
& L_{\text {on }}=\left(\operatorname{Lon}_{\text {in }}-28.85185\right) * 10000
\end{aligned}
$$

Since the decimal degrees of the coordinates are very small, those formulas are used. The fuzzy logic system can easily detect the distance between the measurement positions, by scaling according to a specific position by using the coordinates. The output parameter of the system is the average total electric field value. This value has been entered into the system by taking the minimum and maximum value range of the measurement data.

In the estimation with fuzzy logic, there is a relation between input and output parameters. Table of rules should be created according to the input parameter values in order to get the output parameter values. As a result of this table, an output data is estimated according to the input value.

As an input membership function, the position values are divided into 9 equal parts which are latitude and longitude of the measurement point. Rules have been created with obtained membership functions in order to calculate output values. A total of 16 rules were created to increase the accuracy of the output data.

Fuzzy logic estimation is applied to average electric field values including total average electric field value, GSM, UMTS and LTE frequency bands as in ANN estimation method. However, when the field values for these frequencies are estimated, the input parameters used are latitude and longitude values, in addition, the output values are estimated by entering the maximum and minimum measured value of this frequency as an input.

\section{Results}

Average field values were estimated according to the total average electric field value and frequency. Regression analysis was applied to the results obtained by artificial neural networks. The calculated regression value, $\mathrm{R}^{2}$, is 0.8422 for the total electric field, 0.64913 for GSM, 0.5033 for UMTS and 0.8462 for LTE. These values could be better if more measurements could have been performed. In addition, the mean square error values are calculated in order to analyse the accuracy of the prediction results given by the trained network. In Fig. 4, the MSE values for total EM (Top-Left), for GSM (Top-Right), for UMTS (Bottom-Lleft) and for LTE (Bottom-Right) are given. 



Figure 4: Total EM ANN regression (Top-left), GSM EM ANN regression (Top-right), UMTS EM ANN regression (Bottom-Left), LTE EM ANN regression (Bottom-right).

In addition, the correlation coefficient values of the Measurement-ANN-Fuzzy methods of the total average electric field, GSM average electric field, UMTS average electric field and LTE average electric field values are shown in Table 1, Table 2, Table 3 and Table 4 respectively.

Table 1. Total average E-field correlation coefficient

\begin{tabular}{l|lll}
\hline Method & Fuzzy & Measurement & ANN \\
\hline Fuzzy & 1 & 0.942636 & 0.966254 \\
Measurement & 0.942636 & 1 & 0.921688 \\
ANN & 0.966254 & 0.921688 & 1 \\
\hline
\end{tabular}

Table 2. GSM E-field correlation coefficient

\begin{tabular}{c|ccc}
\hline Method & Fuzzy & Measurement & ANN \\
\hline Fuzzy & 1 & 0.939559 & 0.819398 \\
Measurement & 0.939559 & 1 & 0.805687 \\
ANN & 0.819398 & 0.805687 & 1 \\
\hline
\end{tabular}

Table 3. UMTS E-field correlation coefficient

\begin{tabular}{c|ccc}
\hline Method & Fuzzy & Measurement & ANN \\
\hline Fuzzy & 1 & 0.943614 & 0.65603 \\
Measurement & 0.943614 & 1 & 0.709447 \\
ANN & 0.65603 & 0.709447 & 1 \\
\hline
\end{tabular}


Table 4. LTE E-field correlation coefficient

\begin{tabular}{c|ccc}
\hline Method & Fuzzy & Measurement & ANN \\
\hline Fuzzy & 1 & 0.960722 & 0.90267 \\
Measurement & 0.960722 & 1 & 0.919899 \\
ANN & 0.90267 & 0.919899 & 1 \\
\hline
\end{tabular}

Obtained measurement and estimated electric field values are compared with each other. The measurement points and the electric field values obtained at these points are plotted as $\mathrm{V} / \mathrm{m}$. In comparison, the closest prediction method has been determined with measurement values. The estimated results are compared with the values obtained by the measurements, ANN and fuzzy logic in Fig. 5, Fig.6, Fig. 7 and Fig. 8 respectively.

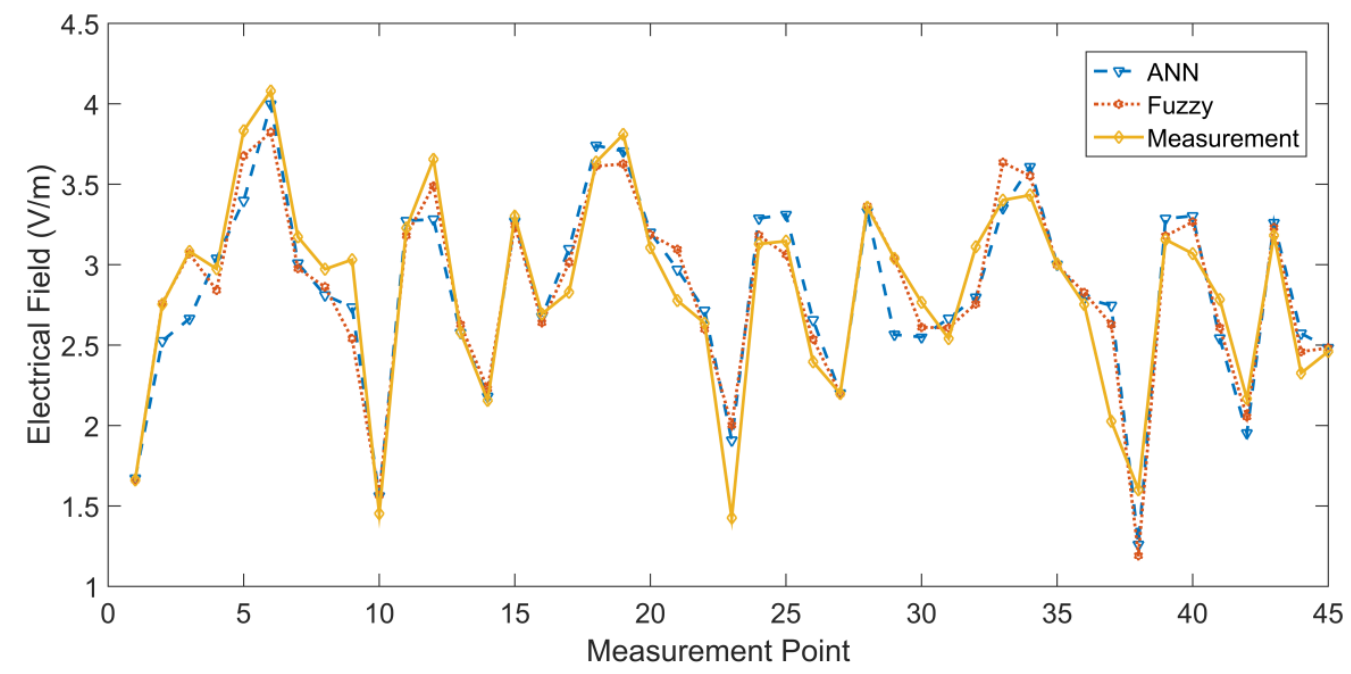

Figure 5. Total average E- Field



Figure 6. GSM E-Field 
When Table 1 and Fig. 5 are analyzed, it is seen that the correlation rate between measurement data and fuzzy logic is $94 \%$, as well as it is $92 \%$ between measurement and ANN. When the results for GSM are examined in Table 2, the similarity is $93 \%$ between measurement data and fuzzy logic and $80 \%$ between measurement and ANN as it is seen in Fig. 6.

For UMTS, there is a 94\% correlation between measurement data and fuzzy in (Table3 and Fig.7) and 70\% between the measurement and ANN. Finally, the graph obtained for LTE shows a correlation of $96 \%$ between measurement and fuzzy and $91 \%$ between measurement and ANN (Table 4 and Fig. 8).

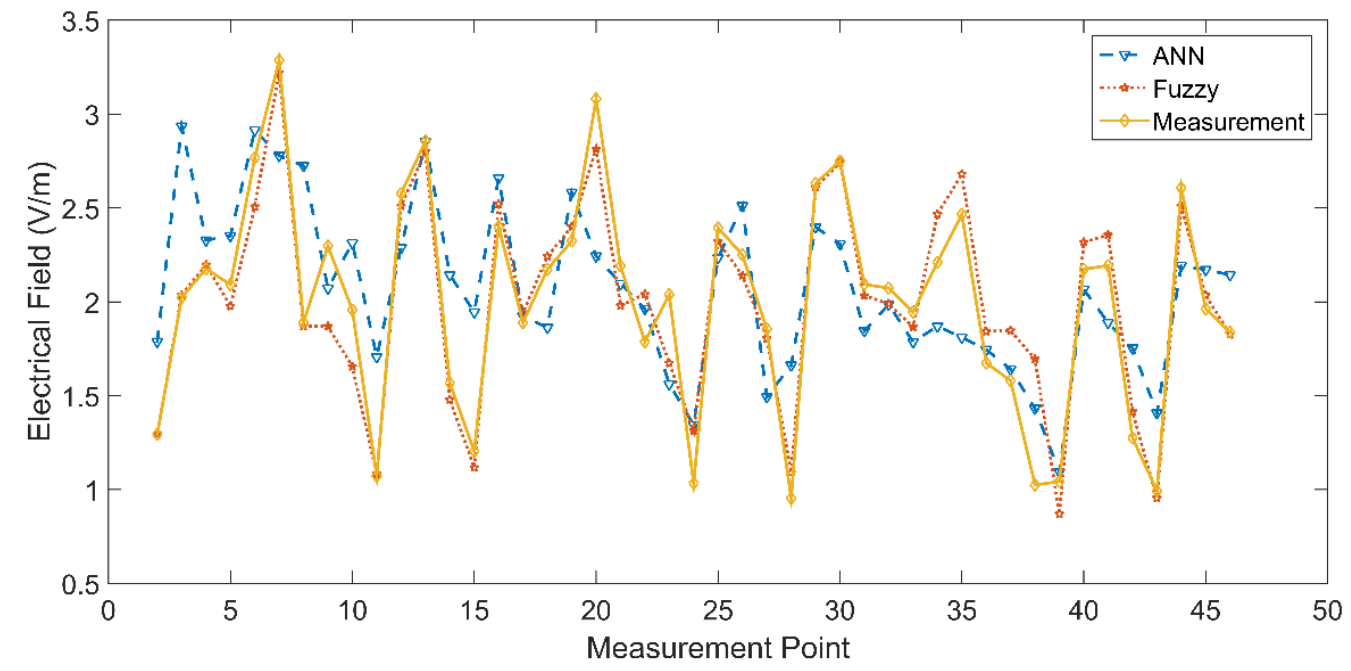

Figure 7. UMTS E-Field

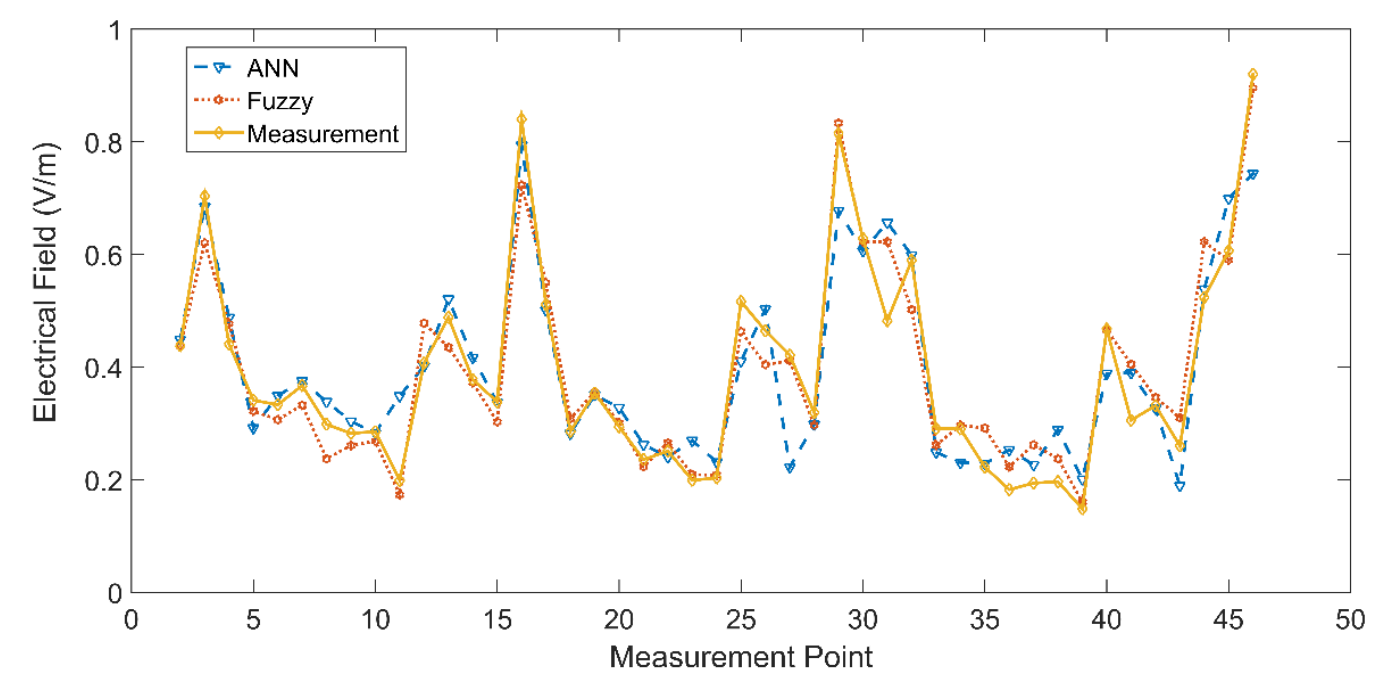

Figure 8. LTE E-Field

\section{Conclusions}

The average electrical field value level of the environment in clinics with medical devices required by national standards should not exceed $3 \mathrm{~V} / \mathrm{m}$ for indoor measurements. As a result of measurements, the highest measured value was found to be $2.88 \mathrm{~V} / \mathrm{m}$ in clinics and the most 
important parameter of signal attenuation was found to be due to the building materials of concrete walls which have 75-80 cm thickness approximately.

When the estimation methods and measurement results are compared, accuracy of fuzzy logic estimation results are higher than ANN estimation results which is determined by the average electric field.

When the obtained data are compared with the measured average results, it is observed that the electric field values change nonlinearly. For this reason, it is possible to obtain better learning of the network by taking more measurements in future studies in order to increase the accuracy of predictions.

In this study, it was determined that the average electric field value within the region can be estimated with latitude and longitude values only in the regions with a certain amount of base stations.

Finally, the measurement and estimation of the electromagnetic pollution, which is a result of $2 \mathrm{G}, 3 \mathrm{G}$ and $4.5 \mathrm{G}$ communication infrastructure are performed and the capability of both ANN and fuzzy logic methods are illustrated.

\section{Acknowledgements}

This Study is supported by the Uludag University AYP (MH2016/1) Scientific Research Project.

\section{References}

[1] Kurnaz, C. (2016). An Empirical Modeling of Electromagnetic Pollution on a University Campus. ACES Express Journal, Vol. 1- 2, pp. 76-79.

[2] Kurnaz, C., Yıldı, D., Karagol, S. (2017). Assessment Of Short/Long Term Electric Field Strength Measurements for a Pilot District. 18th International Symposium Electromagnetic Fields in Mechatronics, Electrical and Electronic Engineering (ISEF) Book of Abstracts.

[3] Mousa, A. (2011). Electromagnetic Radiation Measurements and Safety Issues of Some Cellular Base Stations in Nablus. Journal of Engineering Science and Technology Review, Vol. 4-1, pp. $35-42$.

[4] ICNIRP(2002). Statement General Approach to Protection Against Non Ionizing Radiation, Health Physics 82(4), pp.540-548.

[5] Health and Electromagnetic Fields, https://ec.europa.eu.

[6] ICTA Turkey Electromagnetic Exposure Standards, https://www.btk.gov.tr/en-US/.

[7] ICNIRP Guidelines(1998). Guidelines for Limiting Exposure to Time-Varying Electric, Magnetic, and Electromagnetic Fields (up to 300GHz). International Commission on Non-Ionizing Radiation Protection, Health Physics vol.74, no.4, pp.494-522.

[8] Liechti, R., Stärk, K. D. C., Mobile Communication Base Stations and Animal Health Risk Perception Among Swiss Veterinarians and Farmers. 10th International Symposium on Veterinary Epidemiology and Economics, 2003. 
[9] Ibrani, M., Ahma, L., Hamiti, E., Haxhibeqiri , J.(2011). Derivation of Electromagnetic Properties of Child Biological Tissues at Radio Frequencies. Progress in Electromagnetics Research Letters, Vol. 25, pp. 87-100.

[10] Elliott, P., Toledano, M.B., Beale , L., Hoogh, K., Best, N., Briggs, D.J.(2010). Mobile Phone Base Stations and Early Childhood Cancers: Case-Control Study. BMJ, Vol:22;340:c3077.

[11] Lee, K., Yun, J. (2011). A Comparison of Specific Absorption Rates in SAM Phantom and Child Head Models at 835 and 1900 MHz. IEEE Transactıons On Electromagnetıc Compatibility, Vol. 53, No. 3, pp. 619-627.

[12] Ibrani, M., Ahma, L., Hamiti, E. (2014). Assessment of The Exposure of Children to Electromagnetic Fields from Wireless Communication Devices in Home Environments. IET Communications, Vol. 8-12, pp. 2222-2228.

[13] Everaert, J., Bauwens, D. (2007). A Possible Effect of Electromagnetic Radiation from Mobile Phone Base Stations on the Number of Breeding House Sparrows (Passer domesticus). Electromagnetic Biology and Medicine, Vol. 26, pp. 63-72.

[14] Sorgucu, U., Develi, I.(2012). Measurement and Analysis of Electromagnetic Pollution Generated by GSM-900 Mobile Phone Networks in Erciyes University Turkey. Electromagnetic Biology and Medicine, Vol. 31(4), pp. 404-415.

[15] Peyman, A., Khalid, M., Calderon, C., Addison, D., Mee, Maslanyj, T., M., Mann, S.(2011). Assessment of Exposure To Electromagnetic Fields From Wireless Computer Networks (Wi-Fi) in Schools; Results of Laboratory Measurements. Health Phys., Vol. 100-6, pp. 594-612.

[16] Kurnaz, Ç., Engiz, B.K.(2016). Measurement and Evaluation of Electric Field Strength in Samsun City Center. International Journal of Applied Mathematics, Electronics, and Computers, Vol. 4, pp. 24-29.

[17] Engiz, B. K., Kurnaz, C.(2016). Long-Term Electromagnetic Field Measurement and Assessment for A Shopping Mall. Radiation Protection Dosimetry, pp. 1-9.

[18] Sarolic, A., Matic, P. (2010). Wireless LAN Electromagnetic Field Prediction for Indoor Environment Using Artificial Neural Network. Automatıka, Vol. 51-3, pp. 233-240.

[19] Gül, B. K., Kurnaz, Ç., Engiz, B. K. (2015). Measurement and Evaluation of Electromagnetic Pollution in Ondokuz Mayıs University Kurupelit Campus in Samsun Turkey. Third Intl. Conf. on Advances in Information Processing and Communication Technology - IPCT.

[20] Tuysuz, B., Mahmutoglu, Y. (2017). Measurement and Mapping of the GSM-Based Electromagnetic Pollution in the Black Sea Region of Turkey. Electromagnetic Biology and Medicine, Vol. 36- 2, pp.132-140.

[21] Schmid, G., Lager, D., Preiner, P., Berbacher, R. U., Cecil, S.(2007). Exposure Caused by Wireless Technologies Used for Short-Range Indoor Communication in Homes and Offices. Radiation Protection Dosimetry, Vol. 124-1, pp. 58-62.

[22] Diaz, P. G., Sanz, S. S., Figueras, J.A.P., Fernández, S. J.(2013). Mobile Network Deployment Under Electromagnetic Pollution Control Criterion: An Evolutionary Algorithm Approach. Expert Systems with Applications, Vol. 40, pp. 365-376. 
[23] Perez, P. G., García, M. C., Cuiñas, I., Caldeirinha, R. F.S.(2017). Modeling and Inferring the Attenuation Induced by Vegetation Barriers at 2G/3G/4G Cellular Bands Using Artificial Neural Networks. Measurement, Vol. 98, pp.262-275.

[24] Beekhuizen, J., Vermeulen, R., Eijsden, M. V. , Van Strien, R., Bürgi, A., Loomans,E., Guxens, M., Kromhout, H., Huss, A. (2014). Modelling Indoor Electromagnetic Fields (EMF) from Mobile Phone Base Stations for Epidemiological Studies. Environ Int. Vol. 67, pp. 22-6.

[25] Sadiku, M., Elements of Electromagnetics, Oxford University Press, 2007.

[26] Dianah, A. R. S. N., . Hazmin, S. N., Umar , R., Kamarudin, M. K. A., and Dagang, A. N. (2017), A Review on Electromagnetics (EM) Exposure Measurement Techniques From Base Station, Journal of Fundamental and Applied Sciences, Vol. 9, pp. 182-198 doi:http://dx.doi.org/10.4314/jfas.v9i2s.14

[27] Balanis, C. A., Advanced Engineering Electromagnetics, John Wiley \& Sons, 2012.

[28] Neural Network Toolbox ${ }^{\mathrm{TM}}$ User's Guide, Mathworks, R2017b, http://www.mathworks.com.

[29] Fuzzy Logic Toolbox ${ }^{\mathrm{TM}}$ User's Guide, Mathworks, 2017, http://www.mathworks.com. 